\title{
The cone of curves associated to a plane configuration
}

\author{
C. Galindo* and F. Monserrat**
}

\section{Introduction}

In the last decades, cones associated to varieties have been a basic tool to approach the theory of minimal models. Although this theory works in the case of smooth surfaces by using Castelnuovo criterion to contract $(-1)$-curves, the higher dimensional case is much more difficult. For treating it, there exists a minimal model program, where Kawamata's Theorem [5] on the cone of curves associated to a variety $X$ plays an important role. This theorem generalizes a result by Mori [9] and guarantees that the cone $\overline{N E}(X)$ is rational polyhedral if the anticanonical bundle of $X$ is ample.

On the other hand, the characteristic cone $\tilde{P}(Z / X)$, introduced by Hironaka, is considered in [3] to study projective birational morphisms $\pi: Z \rightarrow X$ which are an isomorphism outside $\pi^{-1}(O)$, for a closed point $O \in X$, where $X$ and $Z$ are normal algebraic varieties over an algebraically closed field. Set $A_{1}(Z / X)$ the $\mathbb{R}$-vector space $N_{1}(Z / X) \otimes_{\mathbb{Z}} \mathbb{R}, N_{1}(Z / X)$ being the commutative group of 1-dimensional cycles on $Z$ which are mapped to $O$ by $\pi$ modulo numerical equivalence and $\mathbb{R}(\mathbb{Z})$ the set of real (integer) numbers. Consider $N E(Z / X)$ the (convex) cone in $A_{1}(Z / X)$ spanned by the cosets of effective curves in $Z$ which are mapped to $O$ by $\pi$. Denote by $A^{1}(Z / X)$ the dual vector space of $A_{1}(Z / X)$ and by $P(Z / X)$ the dual cone of $N E(Z / X)$.

A topological cell of a cone $C$ is defined to be a cone $D$ such that either it is equal to $C$ or it is a maximal cone contained in $E-\operatorname{interior}(E)$, where $E$ is some larger cell of $C$ and interior $(E)$ denotes the relative interior of $E$. It is proved in [6] that a one to one correspondence can be given between sandwiched varieties of $\pi: Z \rightarrow X$ and topological cells of $\tilde{P}(Z / X)$. The relation between topological cells of $\tilde{P}(Z / X)$ and of $P(Z / X)$ shows that if the cone $N E(Z / X)$ is polyhedral, then the set of sandwiched varieties associated to $\pi$ is finite [1]. Recall that sandwiched varieties are those normal schemes through which $\pi$ factorizes by birational projective maps.

Now, assume that $\operatorname{dim} X=3$. Suppose also that $\pi$ is given by a constellation of

\footnotetext{
*Supported by MCyT BMF2001-2251, F. Bancaixa P1-1A2001-03 and by GV048-19.

** Supported by MCyT BMF2001-2251 and by GV048-19.
} 
infinitely near points over $X$, that is, it is given by a configuration of infinitely near points over the variety $X$ - see the Section 2 for the definition - with a unique point at $X$. Denote by $B_{i}$ the exceptional divisor which appears after blowing-up each infinitely near point of the constellation and by $E_{i}$ its strict transform in $Z$. Then, it can be proved [1] that the number of sandwiched varieties associated to $\pi$ is finite provided that every cone $N E\left(E_{i}\right)$ is polyhedral, $N E\left(E_{i}\right)$ being the cone spanned by the images in $A_{1}\left(E_{i}\right)$ of the cosets in $N_{1}\left(E_{i}\right)$ of effective curves on $E_{i}$, where $N_{1}\left(E_{i}\right)$ denotes the commutative group of 1-cycles on $E_{i}$ modulo numerical equivalence and $A_{1}\left(E_{i}\right)$ the $\mathbb{R}$-vector space $N_{1}\left(E_{i}\right) \otimes_{\mathbb{Z}} \mathbb{R}$.

This paper follows the above outlined way, started by Campillo and GonzálezSprinberg in [1] and recently continued in [2], which consists on studying projective birational morphisms by means of cones. It motivates the study of the following problem. Set $X=\mathbb{P}^{2}$ the bidimensional projective space over an algebraically closed field of characteristic zero and $\boldsymbol{K}$ a configuration of infinitely near points over $X$ which gives a projective birational morphism $\pi: Z \rightarrow X$, usually called a modification of $X$. We are interested in the polyhedrality of the cone $N E(Z)$, also called the cone of curves associated to $\boldsymbol{K}$. Notice that, in most cases, the anticanonical bundle of the variety $Z$ is not ample.

There exist other reasons which make interesting the study of the polyhedrality of $N E(Z)$ as Nikulin says in [10]. Those are that surfaces whose cone of curves is polyhedral can be considered as Algebraic Geometry analogue of arithmetic groups generated by reflections in hyperbolic spaces and that it is expected that quantum cohomology of varieties fibrated by surfaces $Z$ with polyhedral cone of curves have good applications, since the set of exceptional curves of $Z$ can be considered as the analogue of a system of simple real roots.

The main goal of this paper is to prove that, roughly speaking, if $\pi$ corresponds to a case singular enough, then the cone $N E(Z)$ is polyhedral.

In the course of this paper, we shall prove that $N E(Z)$ is a polyhedral cone if, and only if, the set of its extremal rays and possibly other ones of $\overline{N E}(Z)$ with null self-intersection has no limit points. These limit points (if they exist) are given by points which are in the intersection between a half-space associated to the canonical divisor class on $Z$ and the unit sphere in an ambient space of dimension equal to the cardinality of the configuration $\boldsymbol{K}$. Moreover, we deduce that the cone of curves of a configuration of cardinal eight or less is always polyhedral (see [8, Theorem 26.2], for the case when all the blown-up points are in $\mathbb{P}^{2}$ ).

To decide the polyhedrality of the cone $N E(Z)$ for configurations of higher cardinality, we give a geometrical condition in Theorem 1 and an explicit one in Theorem 2. The statement of the second referred theorem is simple: The cone of curves is polyhedral whenever $x G x^{t}>0$ for all vector $x \in \mathbb{R}^{n} \backslash\{0\}$ of nonnegative coordinates, where $G$ is an explicit and easy to compute $n$-dimensional square matrix, which depends on the singularity of the configuration (of cardinality $n$ ) $\boldsymbol{K}$. From the study 
of the entries of the matrix $G$, we can conclude that if the singularity of $\boldsymbol{K}$ is large enough, measured in terms of proximity chains among the points in $\boldsymbol{K}$ (see Definition 4), then the cone $N E(Z)$ is polyhedral. The condition established in Theorem 2 can be strengthened when the configuration is a chain (Proposition 6) and so, we can guarantee that the cone $N E(Z)$ is polyhedral only by inspecting the sign of the entry $(n, n)$ in the matrix $G$. Notice that this fact provides a wide range of examples whose associated cone of curves is polyhedral. Finally, we derive a consequence to ensure polyhedrality in the case when $\boldsymbol{K}$ is the configuration associated to a germ of analytically irreducible plane curve.

\section{Preliminaries}

Let $X$ be a smooth variety of dimension $d \geq 2$, we shall consider varieties obtained from $X$ as follows: Take finitely many closed points in $X: Q_{1}^{1}, Q_{1}^{2}, \ldots, Q_{1}^{r}$. Blowup $X$ at $Q_{1}^{1}$ and the obtained variety at $Q_{1}^{2}$ and so on. Denote by $B_{1}^{i}(1 \leq i \leq r)$ the exceptional divisor associated to the blowing-up at $Q_{1}^{i}$. The closed points of $B_{1}^{i}$ $(1 \leq i \leq r)$ are called points in the first infinitesimal neighborhood of $Q_{1}^{i}$. Now, pick finitely many closed points at each divisor $B_{1}^{i}$ and blow-up the last obtained variety at each new point. We can iterate this method finitely many times. For $j>0$, define inductively the points in the $j$ th infinitesimal neighborhood of $Q_{1}^{i}$ as the points in the first infinitesimal neighborhood of some point in its $(j-1)$ th infinitesimal neighborhood. The points $Q$ which are in the $j$ th infinitesimal neighborhood of some point $P$ appearing in the above described process for some $j>0$ are also called infinitely near points to $P$ (this will be denoted $P<Q$ ). A family of closed points as we have described is called to be a configuration $\boldsymbol{K}$ (of infinitely near points over $X$ ) and the obtained variety after the last blowing-up will be called the sky of the configuration and usually denoted by $Z$. Notice that the relation $<$ is a strict partial ordering in $\boldsymbol{K}$. The points $Q_{1}^{i}$ will be said points of level 0 , those at $B_{1}^{i}$ of level 1 and so on. Due to the local character of the blowing-up, we do not need to take into account the order in which the points are blown-up.

We usually denote a configuration by $\boldsymbol{K}=\left\{Q_{1}, Q_{2}, \ldots, Q_{n}\right\}$, bearing in mind that if $Q_{i}<Q_{j}$ then $i<j$. K provides a finite sequence of point blowing-ups, called a modification of $X$ :

$$
Z=X_{n+1} \stackrel{\pi_{n}}{\longrightarrow} X_{n} \longrightarrow \cdots \longrightarrow X_{2} \stackrel{\pi_{1}}{\longrightarrow} X_{1}=X,
$$

$\pi_{i}$ being the blowing-up at $Q_{i}$. Clearly, a point $Q_{j}$ is infinitely near to $Q_{i}$ if $\pi_{i j}\left(Q_{j}\right)=$ $Q_{i}$, where $\pi_{i j}$ is the composition of the maps associated to $\pi, \pi_{i j}: X_{j} \rightarrow X_{i}$. Furthermore, denote by $B_{i}$ the exceptional divisor that we get after blowing-up $X_{i}$ at $Q_{i}$ and by $E_{i}$ (resp., $E_{i}^{*}$ ) the strict (resp., total) transform of $B_{i}$ in $Z$. We shall say 
that $Q_{j}$ is proximate to $Q_{i}$ (denoted by $j \rightarrow i$ or $Q_{j} \rightarrow Q_{i}$ ) whenever $Q_{j}$ belongs to the strict transform of $B_{i}$ in the variety which contains $Q_{j}$.

Set $\boldsymbol{E}=\oplus_{1 \leq i \leq n} \mathbb{Z} E_{i}$, the group of divisors of $Z$ with exceptional support. It is no difficult to see that $E_{i}=E_{i}^{*}-\sum_{j \rightarrow i} E_{j}^{*}$. As a consequence, the set $\left\{E_{i}^{*}\right\}_{1 \leq i \leq n}$ is also a basis of $\boldsymbol{E}$ and the matrix relative to the bases $\left\{E_{i}\right\}$ and $\left\{E_{i}^{*}\right\}$, called the proximity matrix of the configuration $\boldsymbol{K}$, is given by $\left(p_{i j}\right)_{1 \leq i, j \leq n}$, where $p_{i i}=1$, $p_{i j}=-1$ when $i \rightarrow j$ and $p_{i j}=0$, otherwise.

We can associate to each point of a configuration $\boldsymbol{K}$ a nonnegative integer, called its weight or its virtual multiplicity, giving rise to a weighted configuration. Note that weighted configurations are usually called clusters.

Assume that $d=2, \mathcal{K}=\left(\boldsymbol{K},\left\{v_{Q}\right\}_{Q \in \boldsymbol{K}}\right)$ is a weighted configuration and $C$ a curve on $X$. Then we can define the virtual transform of $C$ on $X_{i}$ relative to $\mathcal{K}$ as

$$
\breve{C}_{i}^{\mathcal{K}}=\left(\pi_{1} \circ \pi_{2} \cdots \circ \pi_{i-1}\right)^{*}(C)-\sum_{j=1}^{i-1} v_{Q_{j}} E_{j}^{*},
$$

whenever $2 \leq i \leq n, \breve{C}_{1}^{\mathcal{K}}=C$. The virtual multiplicity of $C$ at $Q_{i}$ relative to $\mathcal{K}$ is defined to be the multiplicity of $\breve{C}_{i}^{\mathcal{K}}$ at $Q_{i}$. We shall say that the curve goes virtually (resp., effectively) through the weighted configuration $\mathcal{K}$ when the virtual transform of $C$ on $Z$ is an effective divisor (resp., it coincides with its strict transform on $Z$ ). We usually say that $C$ goes through $\mathcal{K}$ when it goes virtually through $\mathcal{K}$.

\section{Polyhedrality of the cone of curves}

As we have mentioned in the introduction, set $X=\mathbb{P}^{2}:=\mathbb{P}_{F}^{2}$, where $F$ is an algebraically closed field of characteristic zero. Consider a configuration $\boldsymbol{K}=$ $\left\{Q_{1}, Q_{2}, \ldots, Q_{n}\right\}$ of infinitely near points of $X$ and the associated modification $\pi: Z \rightarrow X$. Denote by $N_{1}(Z)$ the commutative group $\operatorname{Pic}(Z) / \equiv$, where $\equiv$ denotes numerical equivalence and set $A_{1}(Z)=N_{1}(Z) \otimes_{\mathbb{Z}} \mathbb{R}$. Notice that, in our case, $N_{1}(Z)$ is isomorphic to the group of 1 cycles on $Z$ modulo numerical equivalence and that on it, we can consider the intersection form which gives on $A_{1}(Z)$ a bilinear form also denoted by “.”.

Definition 1. We shall define the cone of curves associated to a configuration of infinitely near points of $X, \boldsymbol{K}$, denoted by $N E(Z)$, as the convex cone of $A_{1}(Z)$ spanned by the images in $A_{1}(Z)$ of the cosets in $N_{1}(Z)$ of effective curves on $Z$ modulo numerical equivalence.

Throughout this paper, the numerical equivalence coset in $N_{1}(Z)$ of a divisor $D$ on $Z$ will be denoted by $[D]$ and by an abuse of notation, we usually identify 
an element in $\operatorname{Pic}(Z)$ with its numerical equivalence coset, and also elements (and their intersection form) in $N_{1}(Z)$ with their natural image (and their bilinear form) in $A_{1}(Z)$.

Next, we supply two bases of the $\mathbb{R}$-vector space $A_{1}(Z)$, which we shall use to handle $N E(Z)$. Let $L$ be a projective line on $X$ and let $\bar{L}$ (resp. $L^{*}$ ) be its strict (resp. total) transform on $Z$. Then, it is not difficult to show that $\bar{B}:=\left\{[\bar{L}],\left[E_{1}\right], \ldots,\left[E_{n}\right]\right\}$ and $B^{*}:=\left\{\left[L^{*}\right],\left[E_{1}^{*}\right], \ldots,\left[E_{n}^{*}\right]\right\}$ are bases of $N_{1}(Z)$ as $\mathbb{Z}$-module and, therefore, they are bases of $A_{1}(Z)$ as $\mathbb{R}$-vector space.

We are interested in the polyhedrality of the cone $N E(Z)$. First at all, we study its extremal rays. In what follows, we shall denote by $K$ either the canonical divisor class associated to the variety $Z$ or, by an abuse of notation, its coset modulo numerical equivalence (or, even, its image in $A_{1}(Z)$ ). Moreover, we say that an element in $N_{1}(Z)$ generates a ray of $N E(Z)$ when its image in $A_{1}(Z)$ does so. The following result is an easy consequence of the Riemann-Roch Theorem.

Proposition 1. Let $[D]$ be the coset in $N_{1}(Z)$ of an integral curve $D$ on $Z$ that generates an extremal ray of the cone $N E(Z)$. Then:

i) The intersection number $D \cdot D=D^{2}$ satisfies $D^{2} \leq 0$.

ii) It holds that either $D^{2}<0$ or $K \cdot[D] \geq 0$, whenever $D$ is the strict transform on $Z$ of an integral curve $C$ on $X$ and some point in $\boldsymbol{K}$ does not belong to the strict transform of $C$ on the variety $X_{i}$ containing it.

Remark. An interesting, but obvious, fact is that if $A$ is an effective curve on $Z$, then there exists finitely many integral curves $C$ on $Z$ such that $A \cdot C<0$.

Next, we state some straightforward consequences of the above remark:

Remark. Let $C$ be an integral curve on $Z$ such that $C^{2}<0$. Then:

i) $C$ is the unique integral curve on $Z$ whose coset in $N_{1}(Z)$ generates the ray that it does.

ii) If, in addition, $D$ is an integral curve on $Z$ different from $C$, then the inequality $C \cdot D \geq 0$ holds.

iii) $[C]$ generates an extremal ray of the cone $N E(Z)$.

Furthermore, if $z$ is an extremal ray of the closure of $N E(Z), \overline{N E}(Z)$, such that $z^{2}<0$, then $z$ must also be an extremal ray of $N E(Z)$.

The family $\mathcal{F}=\left\{\left[E_{i}\right]\right\}_{i=1}^{n}$ is a linearly independent set of the $\mathbb{Z}$-module $N_{1}(Z)$. So, each $\left[E_{i}\right]$ gives rise to an extremal ray of the cone $N E(Z)$ because if $\left[E_{i}\right]$ were equal to a linear combination (with nonnegative coefficients) of cosets of irreducible curves on $Z$, this combination would involve only elements in $\mathcal{F}$.

Since Kawamata's Cone Theorem (see [5]) asserts that the set of extremal rays of the cone $N E(Z)$ in the region given by $K \cdot z<0$ is discrete, we are interested 
in studying the region of $N E(Z)$ given by $K \cdot z \geq 0$. It is straightforward, from the remark after Proposition 1 , that if there is a curve of degree $3 l$ which goes (virtually) through the configuration $\boldsymbol{K}$ with multiplicities equal to $l$, then there are finitely many images of irreducible curves of $Z$ in the region given by $K \cdot z>0$.

From now on, fix an ample divisor $H$ on $Z$ and assume that $n \geq 2$ (note that when $n=1$, the cone $N E(Z)$ is polyhedral). For any divisor $D$ on $Z$, set $D(1):=\{z \in$ $\left.A_{1}(Z) \mid[D] \cdot z=1\right\}$ and consider the function

$$
\phi_{D}:\left\{z \in A_{1}(Z) \mid[D] \cdot z>0\right\} \rightarrow D(1),
$$

which maps $z$ to the intersection point between the hyperplane $D(1)$ and the line joining 0 and $z$. Finally, denote by $\overline{N E}(Z)_{Y}$ the set $\overline{N E}(Z) \cap Y$, whenever $Y$ be a subset of $A_{1}(Z)$.

The following definition gives three sets which will be broadly used along this paper.

Definition 2. We shall denote by $\mathcal{R}(\overline{\mathcal{R}}$, resp.) the set of extremal rays of $N E(Z)$ $(\overline{N E}(Z)$, resp.). Also set

$$
\overline{\mathcal{R}}_{0}:=\left\{R \in \overline{\mathcal{R}} \mid r^{2}=0 \text { for all } r \in R\right\} .
$$

Remark. Since $\overline{N E}(Z)$ is a subset of $\mathbb{R}^{n+1}$, we can identify each ray of $\overline{N E}(Z)$ to a point in the unit sphere $S^{n}$ in $\mathbb{R}^{n+1}$. A limit point of $\mathcal{R}, \overline{\mathcal{R}}$ or $\overline{\mathcal{R}}_{0}$ will be the ray generated by a limit point (in $S^{n}$ ) of the set of points in $S^{n}$ that generate rays of the above cited sets. As a consequence of the compactness of $S^{n}$, whichever of the sets $\mathcal{R}, \overline{\mathcal{R}}$ and $\overline{\mathcal{R}}_{0}$ has a no limit point if, and only if, it is finite.

The following result relates the topology of extremal rays to the polyhedrality of the cone $N E(Z)$.

Proposition 2. $N E(Z)$ is a polyhedral cone if, and only if, the sets $\mathcal{R}$ and $\overline{\mathcal{R}}_{0}$ are finite. Furthermore, if this is the case, then $\overline{\mathcal{R}}_{0}$ is empty.

Proof. It suffices to assume that $\mathcal{R}$ and $\overline{\mathcal{R}}_{0}$ are finite. Associated to the ample divisor $H$, we consider the nonnegative half-cone

$$
V=\left\{z \in A_{1}(Z) \mid[H] \cdot z \geq 0 \text { and } z^{2} \geq 0\right\},
$$

which is contained in $\overline{N E}(Z)$ (see [4], V.1.8). By Kleiman ampleness criterion $\overline{N E}(Z)$ is a strongly convex cone and, thus, a system of representatives which generate the rays in $\overline{\mathcal{R}}$ constitutes a minimal set of generators of $\overline{N E}(Z) . \overline{N E}(Z)$ is spanned by the elements of $V$ and the rays in $\mathcal{R}$, and therefore $\overline{\mathcal{R}} \subseteq \mathcal{R} \cup V$. However, $\overline{\mathcal{R}} \cap V=\overline{\mathcal{R}}_{0}$ because those elements that generate rays in $\overline{\mathcal{R}}$ have nonpositive self-intersection. 
Hence, above representatives in $\overline{\mathcal{R}}$ form a finite minimal system of generators of $\overline{N E}(Z)$.

Finally, by Hodge Index Theorem, $V$ is a half-cone over an Euclidean ball of dimension $n$, which is strictly convex. Therefore, $r^{2}<0$ for all generators $r$ of elements of $\overline{\mathcal{R}}$, since $V$ is a subset of $\overline{N E}(Z)$. Then, $\overline{\mathcal{R}} \subseteq \mathcal{R}$ and $N E(Z)$ is a polyhedral cone.

As we have seen, limit points of rays in $\mathcal{R}$ and $\overline{\mathcal{R}}_{0}$ help to decide whether the cone of curves is polyhedral. Therefore we shall give two conditions which must be satisfied by the generators of these limit points. Set $\mathbb{Z}_{+}$the positive integers.

Proposition 3. Let $r \in A_{1}(Z)$ be an element which generates a limit point $R$ of whichever of the sets $\mathcal{R}$ or $\overline{\mathcal{R}}_{0}$. Then $r^{2}=0$ and $K \cdot r \geq 0$.

Proof. The inequality $K \cdot r \geq 0$ follows from the Kawamata's Cone Theorem, since there is no generator of a limit point of the sets $\mathcal{R}$ or $\overline{\mathcal{R}}_{0}$ in the region of $A_{1}(Z)$ given by the inequality $K \cdot z<0$.

It only remains to prove that $r^{2}=0$ when $R$ is a limit point of rays in $\mathcal{R}$. Let $\left\{C_{l}\right\}_{l \in \mathbb{Z}_{+}}$be a sequence of integral curves in $\mathbb{P}^{2}$, such that the cosets in $N_{1}(Z)$ of their strict transforms on $Z,\left[\bar{C}_{l}\right]$, are distinct and whose corresponding rays belong to $\mathcal{R}$ and converge to $R$. Taking coordinates of the $\left[\bar{C}_{l}\right]$ 's in the basis $B^{*}$, we obtain the sequence

$$
\left\{\left[\bar{C}_{l}\right]=\left(d_{l},-e_{l, 1},-e_{l, 2}, \ldots,-e_{l, n}\right)\right\}_{l \in \mathbb{Z}_{+}} .
$$

After normalizing by the first coordinate, we obtain that the ray $r$ will be given by the direction $\left(1,-\lim _{l \rightarrow \infty} \frac{e_{l, 1}}{d_{l}}, \ldots,-\lim _{l \rightarrow \infty} \frac{e_{l, n}}{d_{l}}\right)$.

Now, since for each fixed degree there are finitely many classes in $N_{1}(Z)$ of strict transforms of integral curves in $\mathbb{P}^{2}$, it is clear that the sequence $\left\{d_{l}\right\}_{l=1}^{\infty}$ diverges. Finally, the adjunction formula for the strict transforms of the curves $C_{l}$ proves that

$$
1+\frac{1}{2}\left(d_{l}^{2}-\sum_{i=1}^{n} e_{l, i}^{2}-3 d_{l}+\sum_{i=1}^{n} e_{l, i}\right) \geq 0 .
$$

Dividing by $d_{l}^{2}$ and taking the limit at the infinite, we conclude $r^{2} \geq 0$. Since Proposition 1 proves that $\left[\bar{C}_{l}\right]^{2} \leq 0$, it is clear that $r^{2}=0$.

Remark. With notations as in the above proof, it is clear that the coordinates $\left(e_{l, 1}, e_{l, 2}, \ldots, e_{l, n}\right)$ are the effective multiplicities at the points of the configuration $\boldsymbol{K}$ of the curves $C_{l}$ and so, they satisfy the proximity inequalities $e_{l, i} \geq \sum_{j \rightarrow i} e_{l, j}$, $i=1,2, \ldots, n$ (see [7]). Dividing by $d_{l}$, taking limit at the infinite and setting $r_{i}=\lim _{l \rightarrow \infty} \frac{e_{l, i}}{d_{l}}$, we get that the $r_{i}$ 's also satisfy the proximity inequalities, that is $r_{i} \geq \sum_{j \rightarrow i} r_{j}$. 
Our next result concerns the case when the cardinality of the configuration is small.

Corollary 1. Assume that the cardinality of a configuration $\boldsymbol{K}$ which defines the modification $\pi: Z \rightarrow X$ is $n \leq 9$.

i) If $n \leq 8$, then $N E(Z)$ is polyhedral.

ii) If $n=9$, then either $N E(Z)$ is polyhedral or there is a unique limit point of extremal rays of $N E(Z)$ which is given by $-K, K$ being the canonical divisor class on the variety $Z$. Furthermore, if $\boldsymbol{K}$ has, at least, two points proximate to another third one in $\boldsymbol{K}$, then the cone $N E(Z)$ is polyhedral.

Proof. Set $B:=\left\{z \in \overline{N E}(Z) \backslash\{0\} \mid z^{2}=0\right\}$. i) is a consequence of Propositions 2 and 3 and the fact that, in this case, $B$ is contained in the half-space of $A_{1}(Z)$ given by $K \cdot z<0$ (see the proof of Lemma 1 in [1]).

To prove ii), assume that $N E(Z)$ is not a polyhedral cone. Taking into account that $B \subseteq\left\{z \in A_{1}(Z) \mid\left[L^{*}\right] \cdot z>0\right\}$, we can consider the image of $B$ by $\phi_{L^{*}}$ and so $\overline{\mathcal{R}}_{0}$ has, at most, one point. This follows from Kawamata's Cone Theorem and the fact that, in $\mathbb{R}^{9}$, the hyperplane $\sum_{i=1}^{9} x_{i}=3$ is tangent to the sphere $\sum_{i=1}^{9} x_{i}^{2}=1$ at that point with all its coordinates equal to $1 / 3$. We finish the proof of the first statement by observing that Propositions 2 and 3 show that $\mathcal{R}$ has a unique limit point given by the anticanonical divisor.

Finally, if $\boldsymbol{K}$ has two, or more, points proximate to another third one in $\boldsymbol{K}$, then the cone $N E(Z)$ is polyhedral since, otherwise, the coordinates of the unique limit point of $\mathcal{R}$ must satisfy the proximity inequalities, which is false.

For any subset $S \subseteq A_{1}(Z), \operatorname{Co}(S)$ stands for the convex cone generated by $S$. The following result gives another condition for the cone $N E(Z)$ to be polyhedral.

Theorem 1. The cone $N E(Z)$ is polyhedral if the following condition

$$
\begin{gathered}
\{z \in \overline{N E}(Z) \mid K \cdot z \geq 0\} \cap\left\{z \in \overline{N E}(Z) \mid z^{2}=0\right\} \backslash\{0\} \\
\subseteq \bigcup_{a \in N E(Z)}\left\{z \in A_{1}(Z) \mid a \cdot z<0\right\}
\end{gathered}
$$

holds.

Proof. Proposition 3 and the remark after Proposition 1 show that the set $\mathcal{R}$ has no limit points. We only need to prove that the set $\overline{\mathcal{R}}_{0}$ given at Definition 2 has no limit points.

Suppose that $\overline{\mathcal{R}}_{0}$ has a limit point and look for a contradiction. Let $r$ be a generator of this limit point. It is clear, by Proposition 3, that $K \cdot r \geq 0$ and, from the hypothesis, $[A] \cdot r<0$ for some coset $[A]$ of an effective divisor $A$ on $Z$. 
Let $[A]<0([A] \geq 0$, resp. $)$ denote the half-space of $A_{1}(Z)$ given by $[A] \cdot z<0$ ([A] $z \geq 0$, resp.). Let $T$ be the set of cosets of integral curves in $[A]<0 . T$ is finite by the above mentioned remark.

$N E(Z) \subseteq \operatorname{Co}\left(T \cup \overline{N E}(Z)_{[A] \geq 0}\right)$, because $T$ contains the images in $A_{1}(Z)$ of the integral curves in the half-space $[A]<0$ and $\overline{N E}(Z)_{[A] \geq 0}$ contains the remaining generators. However, $\operatorname{Co}\left(T \cup \overline{N E}(Z)_{[A] \geq 0}\right.$ ) is a closed convex cone (it is generated by a compact set on the hyperplane $H(1))$. Then, $\overline{N E}(Z)=\operatorname{Co}\left(T \cup \overline{N E}(Z)_{[A] \geq 0}\right)$. This implies that the extremal rays of $\overline{N E}(Z)$ in the half-space $[A]<0$ must be generated by elements of $T$ and so we are led to a contradiction to the existence of $r$.

Remark. Next, we state an equivalent condition to that given in the above theorem. It uses the so-called nef cone associated to $Z, P(Z)$. This is the dual cone of $N E(Z)$ with respect to the bilinear form induced by intersection theory. The condition is the following

$$
P(Z) \cap\left\{z \in A_{1}(Z) \mid z^{2}=0\right\} \backslash\{0\} \subseteq\left\{z \in A_{1}(Z) \mid K \cdot z<0\right\},
$$

and the equivalence to the condition in Theorem 1 is an straightforward consequence of the above mentioned fact that the half-cone $V$ given in Proposition 2 is a subset of $\overline{N E}(Z)$.

Corollary 2. The cone $N E(Z)$ associated to a configuration which contains only points on the strict transforms of a conic is polyhedral.

Proof. It suffices to apply Theorem 1 after considering the coset associated with the divisor of the strict transform of the given conic on $Z$.

The next result gives a numerical condition for ensuring that $N E(Z)$ is polyhedral. The proof only considers the virtual transform on $Z$ of a curve $C$ on $X$ relative to a weighted configuration $\mathcal{K}$ and it uses Lagrange multipliers.

Corollary 3. Assume that the cardinality of a configuration $\boldsymbol{K}$ is $n$ larger than 9 , and that a curve $C$ on $\mathbb{P}^{2}$ of degree $d$ goes through a weighted configuration $\mathcal{K}=$ $\left(\boldsymbol{K},\left\{v_{Q_{i}}:=v_{i}\right\}\right)$, such that not all the $v_{i}$ 's are equal. Define

$$
\delta_{j}:=3 \frac{\sum_{i=1}^{n} v_{i}^{2}+\mu_{j} d \sum_{i=1}^{n} v_{i}}{d \sum_{i=1}^{n} v_{i}+n d^{2} \mu_{j}}, \quad j \in\{1,2\},
$$

where $\mu_{j}(j=1,2)$ are the roots of the quadratic equation

$$
d^{2}(9-n) n x^{2}+2 d(9-n) \sum_{i=1}^{n} v_{i} x+9 \sum_{i=1}^{n} v_{i}^{2}-\left(\sum_{i=1}^{n} v_{i}\right)^{2}=0 .
$$

Then $N E(Z)$ is polyhedral, whenever $\min \left\{\delta_{1}, \delta_{2}\right\}>1$. 
Example. Let us take homogeneous coordinates $(X, Y, Z)$ on $\mathbb{P}^{2}$, the point $O=(0,0,1)$ and the standard affine chart of $\mathbb{A}^{2}$ given by $Z \neq 0$. We write $x=X / Z, y=Y / Z$ and consider a configuration $\boldsymbol{K}$ of ten points such that each of these points belongs to the last created divisor and it contains the base points of the ideal $\left(x^{4}, y\right) \mathcal{O}_{\mathbb{P}^{2}}, o$. The quartic $Y^{4}=0$ goes virtually through the weighted configuration $(\boldsymbol{K},\{2,2,2,2,2,2,1,1,1,1\})$. Applying Corollary 3 , we obtain that the cone of curves associated to $\boldsymbol{K}$ is polyhedral, since $\min \left\{\delta_{1}, \delta_{2}\right\} \simeq 1.07>1$.

We desire to give conditions easier to apply which guarantee that the cone of curves associated to a plane configuration $N E(Z)$ is polyhedral. To this purpose, we consider the image of $P(Z) \cap\left\{z \in A_{1}(Z) \mid z^{2}=0\right\} \backslash\{0\}$ by certain map with values in $\mathbb{R}^{n}$ and an explicit cone on $\mathbb{R}^{n}$ that contains it. This fact, jointly the inclusion given in the remark under Theorem 1, will provide the condition asked for.

Let $G$ be a hyperplane in $\mathbb{R}^{n}$ defined by the equation $g(x)=0, x \in \mathbb{R}^{n}$, we shall stand $G^{+}$for the half-space in $\mathbb{R}^{n}$ given by $g(x) \geq 0$.

Definition 3. Let $\boldsymbol{K}=\left\{Q_{1}, Q_{2}, \ldots, Q_{n}\right\}$ and $\pi$ be as above. The convex cone in $\mathbb{R}^{n}$ given by the intersection of the half-spaces $\bigcap_{i=1}^{n} H_{i}^{+}$, where $H_{i}=\left\{x \in \mathbb{R}^{n} \mid\right.$ $\left.x_{i}-\sum_{j \rightarrow i} x_{j}=0\right\}, x=\left(x_{1}, x_{2}, \ldots, x_{n}\right)$, is called proximity cone associated to $\boldsymbol{K}$, $\mathrm{PC}(Z)$.

Next, we obtain explicitly the extremal rays of $\mathrm{PC}(Z)$. Denote $I_{n}:=\{1,2, \ldots, n\}$.

Proposition 4. The extremal rays of the proximity cone $\mathrm{PC}(Z)$ associated to a modification $\pi: Z \rightarrow X$ given by a configuration $K$ are generated by the vectors $e_{k}=\left(e_{1 k}, e_{2 k}, \ldots, e_{n k}\right)(1 \leq k \leq n)$ such that $e_{i k}=0$, whenever $i>k, e_{i i}=1$ and $e_{i k}=\sum_{j \mid k \rightarrow j ; j \geq i} e_{i j}$ if $i<k, i, j, k \in I_{n}$.

Proof. For each $k \in I_{n}$, denote by $L_{k}$ the line on $\mathbb{R}^{n}, L_{k}=\bigcap_{j \neq k} H_{j}$. It is clear that the extremal rays of the cone $\mathrm{PC}(Z)$ are generated by vectors with positive coordinates determined by the lines $L_{k}$. Consider the $(n-1) \times n$ matrices $A_{k}=\left(a_{i j}\right)$ where $i \in I_{n} \backslash\{k\}$ and $j \in I_{n}$, given by $a_{i i}=1, a_{i j}=-1$ when $j \rightarrow i$ and $a_{i j}=0$ otherwise. $L_{k}$ is the solution of the linear system of equations

$$
A_{k} x^{t}=0,
$$

$x=\left(x_{1}, x_{2}, \ldots, x_{n}\right)$ being a variable vector in $\mathbb{R}^{n}$. Set $A_{k}^{D}$ the submatrix of $A_{k}$ gotten by deleting the $k$ th column $a^{k}$ in $A_{k}$. Denote by $b^{k}$ the column vector obtained by deleting the $k$ th coordinate to the vector $-a^{k}$. Thus, the linear system of equations (1) can be written

$$
A_{k}^{D}\left(x_{k}^{D}\right)^{t}=x_{k} b^{k},
$$

where $x_{k}^{D}$ is the variable vector in $\mathbb{R}^{n-1}$ obtained after deleting to $x$ the $k$ th coordinate. Clearly $A_{k}^{D}$ is a regular matrix. Set $\left(A_{k}^{D}\right)^{-1}=\left(s_{i j}\right)_{i, j \in I_{n} \backslash\{k\}}$, then the linear system 
of equations (2) can be expressed $\left(x_{k}^{D}\right)^{t}=x_{k}\left[\left(A_{k}^{D}\right)^{-1} b^{k}\right]$. Whence the vector $x$ is a solution of (1) if and only if for $i \in I_{n} \backslash\{k\}, x_{i}=\left(\sum_{j \mid k \rightarrow j} s_{i j}\right) x_{k}$. Therefore, the equalities $e_{i k}=\sum_{j \mid k \rightarrow j} s_{i j}$ if $i \neq k$ and $e_{k k}=1$ give the coordinates of a generator of the extremal ray relative to $L_{k}$.

It is clear that $e_{i k}=0$ whenever $i>k$. On the other hand, it is straightforward that the entries of the matrix $\left(A_{k}^{D}\right)^{-1}$ satisfy the following relations: $s_{i i}=1, s_{i j}=0$ if $i>j$, and $s_{i j}=\sum_{l \mid j \rightarrow l} s_{i l}$ otherwise. So, for $i<k, e_{i k}=\sum_{j \mid k \rightarrow j} s_{i j}=$ $\sum_{j \mid k \rightarrow j} \sum_{l \mid j \rightarrow l} s_{i l}$. Since the last sum of the righthand of the second equality equals $e_{i j}$ and $e_{i j}=0$, whenever $j<i$, we conclude the proof.

The above given generators of the extremal rays of $\mathrm{PC}(Z)$ will be useful to know when $N E(Z)$ is polyhedral. Therefore, we give an easy way of computing the data $e_{i j}$ which depends on a concept given in the following

Definition 4. Let $\boldsymbol{K}$ be a configuration and $P$ and $R$ points in $\boldsymbol{K}$ such that $P<R$. A proximity chain from $R$ until $P$ is a finite sequence of points in $K,\left\{P_{i}\right\}_{i=0}^{l}$ such that

$$
R=P_{l} \rightarrow P_{l-1} \rightarrow \cdots \rightarrow P_{0}=P .
$$

To understand easily the meaning of each coordinate $e_{i k}$ of the vector $e_{k}$, we can consider the chain of points in the configuration $\boldsymbol{K}$ of the form

$$
Q_{i}=P_{0}<P_{1}<\cdots<P_{l}=Q_{k} .
$$

It is clear that the number of proximity chains in $\boldsymbol{K}$ from $Q_{k}$ until $Q_{i}$ can be computed as the sum of the number of proximity chains until $Q_{i}$ from those points $P$ in the chain such that $Q_{k} \rightarrow P$. Then, proceeding by induction on the length $l$ of the chain (3) and taking into account the formula for $e_{i k}$ given in Proposition 4, we can state the following

Proposition 5. Let $\boldsymbol{K}=\left\{Q_{1}, Q_{2}, \ldots, Q_{n}\right\}$ be a configuration. Then, the coordinate $e_{i k}$ of the generator $e_{k}$ of an extremal ray of the proximity cone $\mathrm{PC}(Z)$ counts the number of proximity chains in $\boldsymbol{K}$ from $Q_{k}$ until $Q_{i}$.

Finally, we state our announced result which gives a condition for the cone $N E(Z)$ to be polyhedral.

Theorem 2. Let $\boldsymbol{K}$ be a configuration of infinitely near points over $X$, which gives a modification $\pi: Z \rightarrow X$. Let $G=\left(g_{l s}\right)$ be the $n \times n$ matrix defined by

$$
g_{l s}=9 \sum_{i=1}^{n} e_{i l} e_{i s}-\left(\sum_{i=1}^{n} e_{i l}\right)\left(\sum_{i=1}^{n} e_{i s}\right),
$$


where $e_{k}=\left(e_{1 k}, e_{2 k}, \ldots, e_{n k}\right)(1 \leq k \leq n)$ are the coordinate vectors that span the extremal rays of the cone $\mathrm{PC}(Z)$ given in Proposition 4. Then, the cone $N E(Z)$ is polyhedral if $x G x^{t}>0$ for all vector $x \in \mathbb{R}^{n} \backslash\{0\}$, such that all its coordinates are nonnegative.

Proof. Consider the sets $U_{0}=\left\{z \in A_{1}(Z) \mid\left[L^{*}\right] \cdot z>0\right\}$ and

$$
Y=\left\{z \in A_{1}(Z) \mid\left[E_{i}\right] \cdot z \geq 0,1 \leq i \leq n\right\},
$$

the homeomorphism $h: L^{*}(1) \rightarrow \mathbb{R}^{n}$, given by $\left(1, x_{1}, \ldots, x_{n}\right) \mapsto\left(-x_{1}, \ldots,-x_{n}\right)$, and the composition map $\mu=h \circ \phi_{L^{*}}$, where $\phi_{L^{*}}$ is the function defined after Proposition 1.

Then, it is clear that $Y \cap U_{0}$ contains $P(Z) \backslash\{0\}$ and $\mu\left(Y \cap U_{0}\right)=\operatorname{PC}(Z)$ (the proximity cone associated to the configuration $K$ ). As a consequence, the following inclusion

$$
\mu\left(P(Z) \cap\left\{z \in A_{1}(Z) \mid z^{2}=0\right\} \backslash\{0\}\right) \subseteq \mathrm{PC}(Z) \cap S^{n-1}
$$

holds, $S^{n-1}$ being the unit sphere in $\mathbb{R}^{n}$. The complement in $\mathbb{R}^{n}$ of the set $\mu(\{z \in$ $\left.\left.A_{1}(Z) \mid K \cdot z<0\right\} \cap U_{0}\right)$ is the set $K^{+}=\left\{\left(x_{1}, \ldots, x_{n}\right) \in \mathbb{R}^{n} \mid \sum_{i=1}^{n} x_{i} \geq 3\right\}$. So, applying the condition given in the remark under Theorem 1, it suffices to check that the set $\operatorname{PC}(Z) \cap S^{n-1} \cap K^{+}$is empty to prove that the cone $N E(Z)$ is polyhedral.

Now, each vector $\alpha=\left(\alpha_{i}\right)_{i=1}^{n}$ in $\mathbb{R}^{n}$ of nonnegative coordinates provides an element in $\operatorname{PC}(Z), \sum_{k=1}^{n} \alpha_{k} e_{k}$, denoted by $r_{\alpha}$. So, the elements in $S^{n-1} \cap \operatorname{PC}(Z)$ are of the form $r_{\alpha} /\left\|r_{\alpha}\right\|$, where $\|\cdot\|$ denotes the norm $\|\cdot\|_{2}$ in $\mathbb{R}^{n}$. Then $N E(Z)$ is polyhedral if

$$
r_{\alpha} /\left\|r_{\alpha}\right\| \in\left\{x \in \mathbb{R}^{n} \mid \sum_{i=1}^{n} x_{i}<3\right\},
$$

for all $\alpha \in \mathbb{R}^{n} \backslash\{0\}$ of nonnegative coordinates. To end the proof, we shall show that the hypothesis of the theorem guarantees the property (4). In fact, $G$ is a symmetric matrix and it defines a quadratic form $g$ which can be expressed by

$$
g(x)=x G x^{t}=9 \sum_{i=1}^{n}\left(\sum_{k=1}^{n} e_{i k} x_{k}\right)^{2}-\left(\sum_{i, k=1}^{n} e_{i k} x_{k}\right)^{2}
$$

and the condition $g(\alpha)>0$ for all vector $\alpha \neq 0$ of nonnegative coordinates proves (4) by taking positive square root, which concludes the proof.

Example. In Figure 1, we depict the proximity graph of a configuration $\boldsymbol{K}$ that satisfies Theorem 2 (see the matrix $G$ below) and so its associated cone $N E(Z)$ is polyhedral. The vertices of the graph represent the points of $\boldsymbol{K}$. Edges join proximate points. An edge joining $P$ and $R(P>R)$ is a continuous straight line whenever $P$ 




Figure 1. The proximity graph of $\boldsymbol{K}$

is in the first infinitesimal neighborhood of $R$, otherwise it is a dotted curved line.

$$
G=\left(\begin{array}{rrrrrrrrrrr}
8 & 7 & 14 & 13 & 12 & 11 & 10 & 9 & 9 & 12 & 11 \\
7 & 14 & 19 & 17 & 15 & 13 & 11 & 9 & 9 & 15 & 13 \\
14 & 19 & 38 & 34 & 30 & 26 & 22 & 18 & 18 & 30 & 26 \\
13 & 17 & 34 & 38 & 33 & 28 & 23 & 18 & 18 & 33 & 28 \\
12 & 15 & 30 & 33 & 36 & 30 & 24 & 18 & 18 & 27 & 21 \\
11 & 13 & 26 & 28 & 30 & 32 & 25 & 18 & 18 & 21 & 14 \\
10 & 11 & 22 & 23 & 24 & 25 & 26 & 18 & 18 & 15 & 7 \\
9 & 9 & 18 & 18 & 18 & 18 & 18 & 18 & 9 & 9 & 0 \\
9 & 9 & 18 & 18 & 18 & 18 & 18 & 9 & 18 & 9 & 0 \\
12 & 15 & 30 & 33 & 27 & 21 & 15 & 9 & 9 & 36 & 30 \\
11 & 13 & 26 & 28 & 21 & 14 & 7 & 0 & 0 & 30 & 32
\end{array}\right) .
$$

Remark. Theorem 2 gives a condition, depending on proximity, that ensures the polyhedrality of the cone of curves associated with a configuration $\boldsymbol{K}$, and this also happens when the cardinality of $\boldsymbol{K}$ is smaller than 9. So, it would be interesting to give an answer, improving that of Theorem 2, to the following question: Given $r \leq 8$ and proximity graphs $\Gamma_{1}, \Gamma_{2}, \ldots, \Gamma_{r}$ of local configurations, when is it true that $N E(Z)$ is polyhedral for any configuration $K$ with points of level $0, P_{1}, P_{2}, \ldots, P_{r}$ and proximity graphs $\Gamma_{1}, \Gamma_{2}, \ldots, \Gamma_{r}$ respectively at $P_{1}, P_{2}, \ldots, P_{r}$ ? 
Next, we shall assume that the configuration $\boldsymbol{K}$ is a chain configuration, that is, each point $Q_{i}$ in $\boldsymbol{K}$ belongs to the divisor created after blowing-up $Q_{i-1}$ for all indices $i$. In this case, we shall show that Theorem 2 provides an easy condition to decide whether the cone $N E(Z)$ is polyhedral. Firstly, we state two supporting results. The first one does not need the configuration to be a chain.

Lemma 1. With notations as in Theorem 2, the elements of the matrix $G=\left(g_{l s}\right)$ are related by the following equalities,

$$
g_{l s}=\sum_{j \mid l \rightarrow j} g_{j s}+9 e_{l s}-\sum_{i=1}^{n} e_{i s} .
$$

Proof. If follows from the following chain of equalities

$$
\begin{aligned}
g_{l s} & =9 \sum_{i=1}^{l-1} e_{i l} e_{i s}+9 e_{l s}-\left(\sum_{i=1}^{l-1} e_{i l}+1\right)\left(\sum_{i=1}^{n} e_{i s}\right) \\
& =9 \sum_{i=1}^{l-1} \sum_{j \mid l \rightarrow j} e_{i j} e_{i s}-\left(\sum_{i=1}^{l-1} \sum_{j \mid l \rightarrow j} e_{i j}\right)\left(\sum_{i=1}^{n} e_{i s}\right)+9 e_{l s}-\sum_{i=1}^{n} e_{i s} \\
& =\sum_{j \mid l \rightarrow j}\left[9 \sum_{i=1}^{l-1} e_{i j} e_{i s}-\left(\sum_{i=1}^{l-1} e_{i j}\right)\left(\sum_{i=1}^{n} e_{i s}\right)\right]+9 e_{l s}-\sum_{i=1}^{n} e_{i s} \\
& =\sum_{j \mid l \rightarrow j} g_{j s}+9 e_{l s}-\sum_{i=1}^{n} e_{i s},
\end{aligned}
$$

where the second equality holds by applying Proposition 4 and the last one is true since $l \rightarrow j$ implies $j<l$.

Lemma 2. Let $\boldsymbol{K}$ be a chain configuration and $G=\left(g_{l s}\right)$ the matrix associated to $\boldsymbol{K}$ given in Theorem 2. If $g_{n n}>0$, then all the entries of the matrix $G$ are positive.

Proof. We shall reason by contradiction. For each index $s(1 \leq s \leq n)$, define $\triangle_{s}=\left\{i \in\{1, \ldots, n\} \mid g_{i s} \leq 0\right\}$ and assume that $\triangle_{s} \neq \emptyset$ for some fixed index $s$. Consider $i_{0}$ the minimum element in $\triangle_{s}$. In the proof, we shall use the following two properties which are easily deduced from the formula that Lemma 1 gives for the element $g_{i_{0} s}$ (which, we know that it is not positive).

- Property 1 . If the point $Q_{i_{0}}$ is proximate to $Q_{k}$ then,

$$
g_{k s}+9 e_{i_{0} s}-\sum_{i=1}^{n} e_{i s} \leq 0 .
$$


- Property 2. $9 e_{j s}-\sum_{i=1}^{n} e_{i s} \leq 0$ for all $j \geq i_{0}$.

Notice that Property 2 holds since it is true for $j=i_{0}$ by Lemma 1 and moreover $e_{j s} \leq e_{i s}$ if $j \geq i$.

Now, we shall prove that $g_{j s} \leq 0$ for all $j \geq i_{0}$. It shows that $g_{n s} \leq 0$ and this will conclude the proof since if $s=n$ we are led to a contradiction and otherwise $g_{s n} \leq 0$ because $G$ is a symmetric matrix and thus the same procedure for $n$ instead $s$ proves $g_{n n} \leq 0$ which is a contradiction.

We can assume that $i_{0}<n$ and, for proving the above inequalities, we shall use the following inductive procedure: First, we shall prove the basic step, that is $g_{i_{0}+1, s} \leq 0$, and after the inductive step, where we shall show $g_{l+1, s} \leq 0$ whenever $g_{l, s} \leq 0$ for all positive integer $j$ such that $i_{0}+1 \leq j \leq l$.

To do the basic step, we distinguish two cases: Case 1 which occurs when there exists an index $k\left(1 \leq k<i_{0}<n\right)$ such that the point $Q_{i_{0}+1}$ is proximate to $Q_{k}$ (and obviously, $Q_{i_{0}}$ is also proximate to $Q_{k}$ ) and the complementary of Case 1 , which we shall refer as Case 2.

In Case 1 we get,

$$
g_{i_{0}+1, s}=g_{k s}+g_{i_{0} s}+9 e_{i_{0}+1, s}-\sum_{i=1}^{n} e_{i s} \leq g_{k s}+g_{i_{0} s}+9 e_{i_{0}, s}-\sum_{i=1}^{n} e_{i s} \leq 0 .
$$

And in Case 2,

$$
g_{i_{0}+1, s}=g_{i_{0} s}+9 e_{i_{0}+1, s}-\sum_{i=1}^{n} e_{i s} \leq 0 .
$$

In both cases the equality is given by Lemma 1 . In Case 1, the first inequality holds since $\boldsymbol{K}$ is a chain configuration. Finally, the fact $g_{i_{0} s} \leq 0$ and the above given Property 1 (resp., 2) for the Case 1 (resp., 2) conclude the proof of the basic step.

Finally, we show the inductive step. Suppose $g_{j s} \leq 0$ for $i_{0}<j \leq l<n$, we shall see that $g_{l+1, s} \leq 0$. Here, we need to distinguish three cases:

i) There exists an index $k\left(1 \leq k<i_{0}<n\right)$ such that the point $Q_{l+1}$ is proximate to $Q_{k}$ (in such case the point $Q_{i_{0}}$ is also proximate to $Q_{k}$ ). Then,

$$
g_{l+1, s}=g_{k s}+g_{l s}+9 e_{l+1, s}-\sum_{i=1}^{n} e_{i s} \leq g_{l s}+g_{k s}+9 e_{i_{0} s}-\sum_{i=1}^{n} e_{i s} \leq 0 .
$$

ii) There exists an index $k\left(1 \leq i_{0} \leq k<l\right)$ such that the point $Q_{l+1}$ is proximate to $Q_{k}$. Then,

$$
g_{l+1, s}=g_{k s}+g_{l s}+9 e_{l+1, s}-\sum_{i=1}^{n} e_{i s} \leq 0
$$


iii) The point $Q_{l+1}$ is only proximate to $Q_{l}$. Then,

$$
g_{l+1, s}=g_{l s}+9 e_{l+1, s}-\sum_{i=1}^{n} e_{i s} \leq 0 .
$$

This ends the proof by noticing that we have applied Lemma 1 in all cases, Property 1 in case i) and Property 2 in cases ii) and iii), and the inductive hypothesis in all cases which asserts that $g_{l s} \leq 0$ in cases i) and iii), and that $g_{k s} \leq 0$ and $g_{l s} \leq 0$ in case ii).

We have obtained an interesting consequence for the associated matrix to chain configurations $\boldsymbol{K}$ : The condition $x G x^{t}>0$ for all vector $x \in \mathbb{R}^{n} \backslash\{0\}$, with nonnegative coordinates, is equivalent to the fact $g_{n n}>0$. Thus, we have proved the following

Proposition 6. Let $\boldsymbol{K}$ be a chain configuration whose associated date $g_{n n}$ given in Theorem 2 is strictly positive. Then, the cone of curves $N E(Z)$ relative to $\boldsymbol{K}$ is polyhedral.

Finally, we state some consequences of Proposition 6, which allow to conclude that the statement on this proposition is not trivial.

Corollary 4. Let $\boldsymbol{K}=\left\{Q_{1}, \ldots, Q_{n}\right\}$ be a chain configuration and let $B$ be a germ of analytically irreducible plane curve which goes through the points in $\boldsymbol{K}$ with effective multiplicities $m_{1}, \ldots, m_{n}$ satisfying the proximity equalities and $9 \sum_{i=1}^{n} m_{i}^{2}-$ $\left(\sum_{i=1}^{n} m_{i}\right)^{2}>0$. Then, the cone of curves associated to $\boldsymbol{K}$ is polyhedral.

Proof. It follows from the fact that the vector of effective multiplicities of $B$ is a multiple of the vector $e_{n}$ in Theorem 2, because it determines the only direction satisfying the proximity equalities. So, the condition given in the statement of the corollary on the multiplicities $m_{i}$ implies $g_{n n}>0$ and the result.

Corollary 5. Let $O$ be a closed point of $\mathbb{P}^{2},\{x, y\}$ local coordinates at $O$ and $\boldsymbol{K}=\left\{Q_{1}=O, Q_{2}, \ldots, Q_{n}\right\}$ the chain configuration corresponding to the minimal embedded resolution of an analytically irreducible germ of plane curve at $O$ with a unique characteristic pair $\left(\beta_{0}, \beta_{1}\right)$. Then, the cone of curves associated to the configuration $\boldsymbol{K}$ is polyhedral if the pair $\left(\beta_{0}, \beta_{1}\right)$ satisfies one of the following conditions:

(1) $\beta_{1} \equiv 1\left(\bmod \beta_{0}\right), \beta_{0} \leq 8$ and $\beta_{1}<8 \beta_{0}$.

(2) $\beta_{1} \equiv 1\left(\bmod \beta_{0}\right), \beta_{0} \geq 9$ and $\beta_{1}<7 \beta_{0}$.

(3) $\beta_{1} \not \equiv 1\left(\bmod \beta_{0}\right)$ and $\beta_{1}<1+\frac{7}{2} \beta_{0}+\frac{3}{2} \sqrt{4 \beta_{0}+5 \beta_{0}^{2}}$. 
Proof. Let $(\overbrace{w_{1}, \ldots, w_{1}}^{k_{1} \text { times }}, \overbrace{w_{2}, \ldots, w_{2}}^{k_{2} \text { times }}, \ldots, \overbrace{w_{s}, \ldots, w_{s}}^{k_{s} \text { times }})$ be the sequence of multiplicities of the germ at the points of the configuration $\boldsymbol{K}$. Then,

$$
w_{1}=\beta_{0}, \quad k_{1}=\left\lfloor\frac{\beta_{1}}{\beta_{0}}\right\rfloor, \quad w_{2}=\beta_{1}-\left\lfloor\frac{\beta_{1}}{\beta_{0}}\right\rfloor \beta_{0}, \quad w_{s}=1,
$$

where $\lfloor\cdot\rfloor$ means the floor (or the integer part) function, and clearly one gets the following recurrence relations:

$$
w_{s-1}=k_{s}, \quad w_{i}=k_{i+1} w_{i+1}+w_{i+2} \quad(1 \leq i \leq s-2) .
$$

By Corollary 4, for the cone of curves associated to $\boldsymbol{K}$ to be polyhedral, we only need to check when

$$
9 \sum_{i=1}^{s} k_{i} w_{i}^{2}-\left(\sum_{i=1}^{s} k_{i} w_{i}\right)^{2}>0
$$

To do it, we distinguish two cases:

i) $\beta_{1} \equiv 1\left(\bmod \beta_{0}\right)$. Here, $s=2, k_{2}=\beta_{0}$ and, then, the condition (7) is equivalent to the following one:

$$
-\beta_{0}\left\lfloor\frac{\beta_{1}}{\beta_{0}}\right\rfloor^{2}+7 \beta_{0}\left\lfloor\frac{\beta_{1}}{\beta_{0}}\right\rfloor+9-\beta_{0}>0,
$$

which is true if, and only if, $\beta_{0}$ and $\beta_{1}$ satisfy the formulae in 1 or 2 of the statement.

ii) $\beta_{1} \neq \equiv 1\left(\bmod \beta_{0}\right)$. By using the conditions $(6)$, one gets that $(7)$ is equivalent to the following inequality:

$$
9\left(w_{1} w_{2}+k_{1} w_{1}^{2}\right)-\left(w_{1}+w_{2}+k_{1} w_{1}-1\right)^{2}>0,
$$

and by means of the equalities (5), this inequality is true if, and only if,

$$
9 \beta_{0} \beta_{1}-\left(\beta_{0}+\beta_{1}-1\right)^{2}>0,
$$

which happens only when the formula in 3 of the statement holds.

Corollary 6. Let $\boldsymbol{K}=\left\{Q_{1}, \ldots, Q_{n}\right\}$ a chain configuration whose proximity graph is that of the following figure with $g \geq 1$ dotted curved lines (its Dynkin diagram has $g$ stars). Then, the cone of curves associated to $\boldsymbol{K}$ is polyhedral.

Proof. It follows from Proposition 6 since the vector $\left(e_{1 n}, \ldots, e_{n n}\right)$ corresponding to this configuration is $\left(2^{g}, 2^{g-1}, 2^{g-1}, \ldots, 2,2,1,1\right)$, where $g=(n-1) / 2$.

Acknowledgment. The authors express their appreciation for a very careful and thorough critique by the referee. 


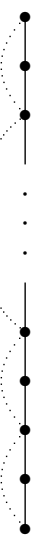

Figure 2. Proximity graph of Corollary 6

\section{References}

[1] A. Campillo, G. González-Sprinberg, On characteristic cones, clusters and chains of infinitely near points. In Singularities (Oberwolfach, 1996), Progr. Math. 162, Birkhäuser, Basel 1998, 251-261. Zbl 0931.13017 MR 1652477

[2] A. Campillo, O. Piltant, A. Reguera, Cones of curves and of line bundles on surfaces associated with curves having one place at infinity. Proc. London Math. Soc. 84 (2002), 559-580. Zbl pre01819266 MR 1888423

[3] S. D. Cutkosky, Complete ideals in algebra and geometry. In Commutative algebra: syzygies, multiplicities, and birational algebra, Contemp. Math. 159, Amer. Math. Soc., Providence, RI, 1994, 27-39. Zbl 0811.13001 MR 1266177

[4] R. Hartshorne, Algebraic Geometry. Grad. Texts in Math. 52, Springer-Verlag, New York, Heidelberg, Berlin 1977, 8th printing 1997. Zbl 0531.14001 MR 0463157

[5] Y. Kawamata, The cone of curves of algebraic varieties. Ann. of Math. (2) 119 (1984), 603-633. Zbl 0544.14009 MR 0744865

[6] S. Kleiman, Towards a numerical theory of ampleness. Ann. of Math. (2) 84 (1966), 293-344. Zbl 146.17001 MR 0206009

[7] J. Lipman, Proximity inequalities for complete ideals in two-dimensional regular local rings. In Commutative algebra: syzygies, multiplicities, and birational algebra, Contemp. Math. 159, Amer. Math. Soc., Providence, RI, 1994, 293-306. Zbl 0814.13016 MR 1266187

[8] Y. Manin, Cubic forms. Algebra, Geometry, Arithmetic. North-Holland Mathematical Library 4, North-Holland, Amsterdam, London 1974. Zbl 0277.14014 MR 0460349

[9] S. Mori, Threefolds whose canonical bundles are not numerically effective. Ann. of Math. (2) 116 (1982), 133-176. Zbl 0557.14021 MR 0662120 
[10] V. V. Nikulin, A remark on algebraic surfaces with polyhedral Mori cone. Nagoya Math. J. 157 (2000), 73-92. Zbl 0958.14026 MR 1752476

Received January 28, 2002; revised April 22, 2003

C. Galindo, F. Monserrat, Departamento de Matemáticas (ESTCE), UJI, Campus Riu Sec., 12071 Castellón, Spain

E-mail: galindo@nuvol.uji.es; monserra@mat.uji.es 